\title{
AIDS Effects in Liver and Biliary Tract by using Ultrasound in Sudan
}

\author{
Mustafa Z Mahmoud ${ }^{1,2}$, Alsafi A Abdulla ${ }^{3}$,Ala M Abd Elgyoum ${ }^{4}$ \\ ${ }^{I}$ Salman bin Abdul-Aziz University, College of Applied Medical Science, Radiology and \\ Medical Imaging Department, Al-Kharj, Saudi Arabia. \\ ${ }^{2}$ Sudan University of Science and Technology, College of Medical Radiological Science, \\ Fundamental Medical Radiologic Sciences Department, Khartoum, Sudan. \\ ${ }^{3}$ Sudan University of Science and Technology, College of Medical Radiological Science, \\ Radiotherapy Department, Khartoum, Sudan. \\ ${ }^{4}$ Alzaeim Alazhari University, Faculty of Radiological Sciences and Medical Imaging, \\ Khartoum North, Sudan.
}

Corresponding Author Mustafa Z. Mahmoud

Mobile:

00966549332852

E mail:

zuhairmustafa4@hotm ail.com.

Received : 12 /8 /2012 Accepted after revision: $15 / 10 / 2012$

Key words: AIDS; Cholecystitis; Hepatomegally; Kaposi sarcoma; Ultrasound
Background and study aim: The human immunodeficiency virus (HIV) is a retrovirus that infects cells of the immune system, destroying or impairing their function. As the infection progresses, the immune system becomes weaker, and the person becomes more susceptible to infections. The United Nations (UN) estimates that Sudan has the highest rate of HIV infection in North Africa and the Middle East. The aim of this study was to describe the effects of AIDS in liver and biliary tract and evaluate the clinical utility of hepatic and biliary tract sonography in AIDS patients in Khartoum state, Sudan.

Patient and Methods: This prospective study was conducted at Omdurman, Elshaab and Bashayer Teaching Hospitals, in Khartoum State, Sudan. It spanned a period of 3 years from January 2009 to January 2012, involving 300 HIV positive Sudanese patients (198; 66\% males and 102; $34 \%$ females) and aged 6 to 60 years; mean age of $46 \pm 6.4$ years.

\section{INTRODUCTION}

Human immunodeficiency virus infection/Acquired immunodeficiency syndrome (HIV/AIDS) is a disease of the human immune system caused by the human immunodeficiency virus (HIV) [1]. The illness interferes with the immune system, making people with AIDS much more likely to get infections, including opportunistic infections and tumors that do not usually affect people with working immune systems. This susceptibility increases as the disease worsens [2].
Samples proved to have AIDS by enzyme immuno assay test (EIA) and also confirmed by Western blot (protein immunoblot) blood test. Sonography was performed using Toshiba Just Vision 200, Aloka SSD 500 and Siemens Antares fitted with $3.5 \mathrm{MHz}$ convex transducers.

Results: In AIDS patients, ultrasound findings in liver included wide spectrum of abnormalities like hepatomegally (88; $29.3 \%$ ), portal hypertension (49; 16.3\%), periportal fibrosis $(20 ; 6.7 \%)$, fatty fibrotic changes $(42 ; 14 \%)$, jaundice (18; $6 \%$ ), extra hepatic ducts dilatation (24; $8 \%)$, cholecystitis (33; $11 \%)$, cholelithiasis $(24 ; 8 \%)$ and Kaposi sarcoma $(18 ; 6 \%)$.

Conclusion: Abdominal ultrasound is a simple and cost effective tool and can be used as a baseline imaging modality in AIDS infected patients. Liver enlargement and gall bladder wall thickening are common effects develop due to AIDS infection in population at Khartoum state, Sudan.

Patients with AIDS present a wide variety of clinical manifestations through involvement of various organs. Ultrasonography is easy to perform, safe, inexpensive, not invasive and repeatable. Ultrasound can investigate most of the organs affected in AIDS and can guide biopsies, allowing the cytohistological and microbiological investigations need for a definitive diagnosis [3]. 
The aim of this study was to describe the effects of AIDS in liver and biliary tract and evaluate the clinical utility of liver and biliary tract sonography in AIDS patients in Khartoum state, Sudan. Despite the fact that the epidemiological data is limited, also absence of any data determines the sonographic findings of AIDS in various body organs of the affected samples.

Ultrasound findings in patients with AIDS were studied by Langer et al. where ultrasonographic findings of 43 patients with AIDS and ARC were analyzed. In $63 \%$ an enlarged liver, in $66 \%$ an enlarged spleen, partially with focal lesions, and in $21 \%$ enlarged abdominal lymph nodes were diagnosed [4]. Non-Hodgkin's lymphomas (NHL) are the second most frequent malignancies in AIDS patients. The majority of NHL associated with AIDS involves extra nodal sites, especially the digestive tract and the central nervous system. Primary liver lymphoma (PLL) is an uncommon neoplasm among these patients. Ultrasonography and computed tomography scans may be helpful in the diagnosis of focal hepatic lymphoma [5].

AIDS was most prevalent in the $4^{\text {th }}$ decade with an incidence of $40.4 \%$ compared with the HIV negative individuals. Ultrasonography is optimally suited for its clinical management especially in Africa. Its accuracy and sensitivity may be much improved with clinico-pathologic correlation which may not be readily available in developing countries; further studies may provide this much needed diagnostic algorithms [6]. Ultrasonographic screening for liver enlargement associated with opportunistic infections in patients with human immunodeficiency virus infection revealed that liver enlargement in $63.75 \%$ of HIV patients. In $40.7 \%$ the right lobe size varied from $140 \mathrm{~mm}$ to $160 \mathrm{~mm}$. Of those with hepatomegally, $60.7 \%$ had AIDS. Liver enlargement is common in HIV infected patients mostly in association with hepatitis C and B viruses and Mycobacterium tuberculosis [7].

Abdominal ultrasonography and computed tomography were performed in two patients with acquired immunodeficiency syndrome (AIDS) and necropsy proved hepatic Kaposi sarcoma. At ultrasound, small $(5-12 \mathrm{~mm})$ hyperechoic nodules and dense periportal bands were seen in the liver. These lesions appeared hypo attenuated on baseline and dynamic CT scans and enhanced on delayed scans after a bolus injection of contrast material. Although nonspecific, these features strongly suggest tumor involvement in the liver in patients with AIDS and Kaposi sarcoma [8].

\section{PATIENTS AND METHODS}

Abdominal ultrasound scans were prospectively performed over a 3 years period (January 2009 to January 2012), involving 300 HIV positive Sudanese patients (aged 6 to 60 years; mean age of $46 \pm 6.4$ years) selected from the outflow of patients at Omdurman Teaching Hospital, Elshaab Teaching Hospital and Bashayer Teaching Hospital, Radiology and Medical Imaging Department, Khartoum State, Sudan. At inclusion, complete medical history, routine clinical and diagnostic procedures including an ultrasound examination of the abdomen, and standard enzyme immuno assay test (EIA) was used to check for antibodies to HIV. If this antibody test states that sample is positive, a confirmatory blood test; Western blot (protein immunoblot) test performed in all patients. The medical records of such 300 qualified patients were analyzed with regard to age, sex, demographic data, liver ultrasound findings, medical history and symptoms. Other samples, which do not apply to them these features, were excluded from the study. An informed consent was obtained from all the subjects before scanning but, in addition, a review and authorization of the study protocols was done by the Ethical Committees available at the Radiology and Medical Imaging Departments of Omdurman, Elshaab and Bashayer Teaching Hospitals. Abdominal sonography was performed using Toshiba Just Vision 200, Aloka SSD 500 and Siemens Antares fitted with 3.5 $\mathrm{MHz}$ convex array transducers. For sonograms printing, ultrasound machines used were connected with digital graphic printer, $100 \mathrm{~V} ; 1.5$ $\mathrm{A}$; and $50 / 60 \mathrm{~Hz}$, with serial number of 3-619GBI-01 and made by Sony Corporation- Japan. All measurements were obtained by electronic calipers available in the ultrasound machines. Prior to exam, Patients should be subjected to abdominal preparation to avoid gas buildup in the intestine. Patient should take nothing by mouth for 8 hours. If fluid is essential to prevent dehydration only water should be given. In case of acute symptoms, examination was proceeding immediately. Infants' clinical condition, permitting was given for nothing by mouth for 3 hours preceding the examination. For most ultrasound exams, the patient is positioned lying 
face-up (supine) on an examination table that can be tilted or moved. A clear water based gel is applied to the abdomen to help the transducer make secure contact with the body and eliminate air pockets between the transducer and the skin that can block the sound waves from passing into patient body. The highest frequency transducer permitting adequate penetration is used such as phased array sector probe with its small footprint permits subcostal and intercostal scanning. Scans were performed in the sagittal and transverse planes from the anterior subcostal and intercostal approach to study the liver and biliary tract to diagnose the various findings related to AIDS effects. Various maneuvers may enhance demonstration of the liver and biliary tract including left posterior oblique left lateral decubitus or setting semierect to erect lateral oblique positions. Coronal right lateral subcostal and intercostal approach with different breathing technique is recommended depending on liver shape and patient respiration [9,10]. The procedures followed were in accordance with the ethical standards of the responsible committee on human experimentation (institutional or regional) and with the Helsinki Declaration of 1964. Results statistical analysis, overviewed in a form of tables and graphs by using Microsoft Office Excel package depend on the frequencies and the percentages of categorical variables of the scanned samples.

\section{RESULTS}

A total of 300 HIV positive Sudanese patients (198; 66\% males and 102; 34\% females) aged from 6 to 60 years; mean age of $46 \pm 6.4$ years, proved to have AIDS by enzyme immuno assay test (EIA) and also confirmed by Western blot (protein immunoblot) blood test. In the sample, AIDS is common in the age group 41 to 50 years; $4^{\text {th }}$ decade with a frequency of (229; 76.3\%) (Table 1 and Figure 1).

Table 1: Age group distribution in AIDS patients

\begin{tabular}{|c|c|c|}
\hline Age group (year) & Frequency & Percentage (\%) \\
\hline < Than 20 years & 2 & $(0.7 \%)$ \\
\hline $\mathbf{2 0 - 3 0}$ & 18 & $(6 \%)$ \\
\hline $\mathbf{3 1 - 4 0}$ & 24 & $(8 \%)$ \\
\hline $\mathbf{4 1 - 5 0}$ & 229 & $(76.3 \%)$ \\
\hline $\mathbf{5 1 - 6 0}$ & 27 & $(9 \%)$ \\
\hline Total & 300 & $100 \%$ \\
\hline
\end{tabular}

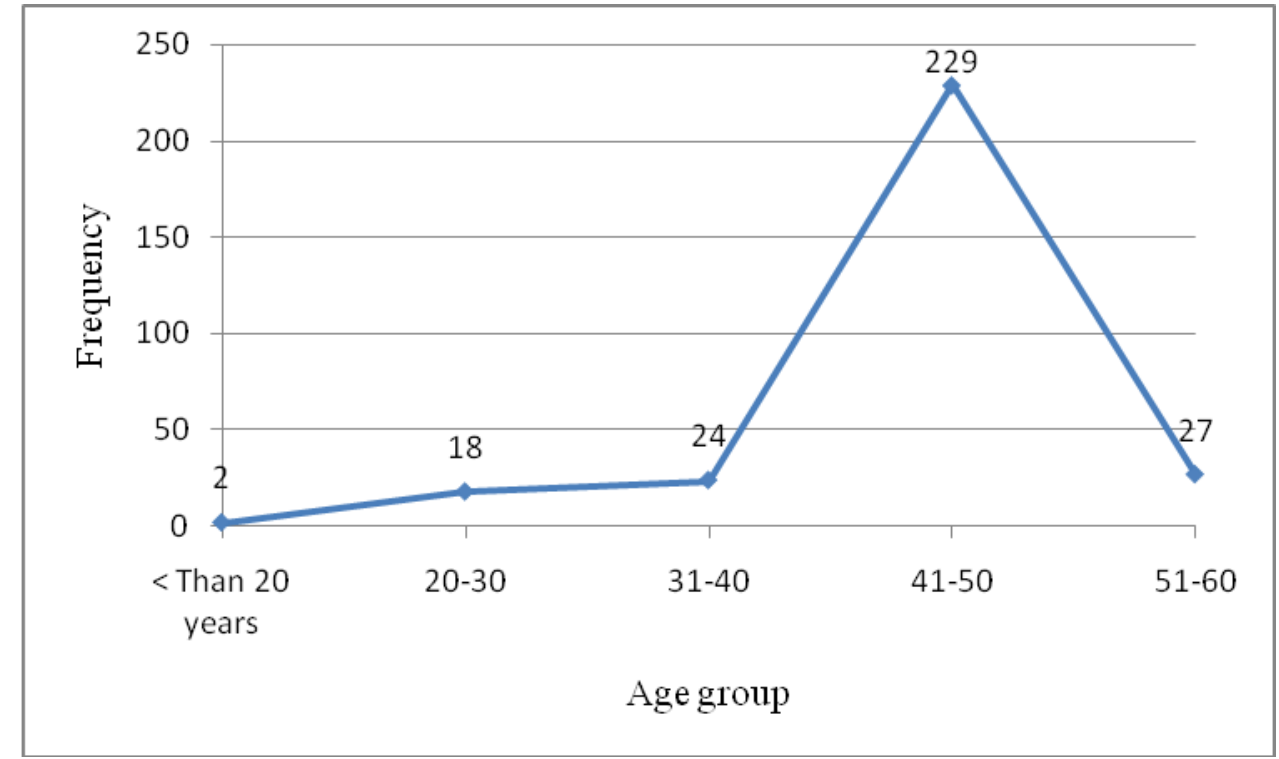

Figure 1: Age group distribution in AIDS patients

The duration of acquired HIV infection is varied with mean of $7.5 \pm 3.8$ years in the sample. Where $(15 ; 5 \%)$ cases were diagnosed in the time period of 2000 to 2002; HIV positive for 12 to 10 years, $(17 ; 5.7 \%)$ cases were diagnosed in the time period of 2003 to 2005; HIV positive for 9 to 7 years and $(268 ; 89.3 \%)$ cases were diagnosed in the time period of (2006 to 2012); HIV positive for 6 to 1 years (Table 2 and Figure 2). 
Table 2: Duration of acquired AIDS infection

\begin{tabular}{|c|c|c|c|}
\hline Date of diagnosis & HIV positive duration & Frequency in patients & Percentage (\%) \\
\hline $\mathbf{2 0 0 0 - 2 0 0 2}$ & 12-10 years & 15 & $(5 \%)$ \\
\hline $\mathbf{2 0 0 3 - 2 0 0 5}$ & 9-7 years & 17 & $(5.7 \%)$ \\
\hline $\mathbf{2 0 0 6 - 2 0 1 2}$ & 6-1 years & 268 & $(89.3 \%)$ \\
\hline Total & 12 years & 300 & $(100 \%)$ \\
\hline Mean \pm SD & $7.5 \pm 3.8$ years & - & - \\
\hline
\end{tabular}

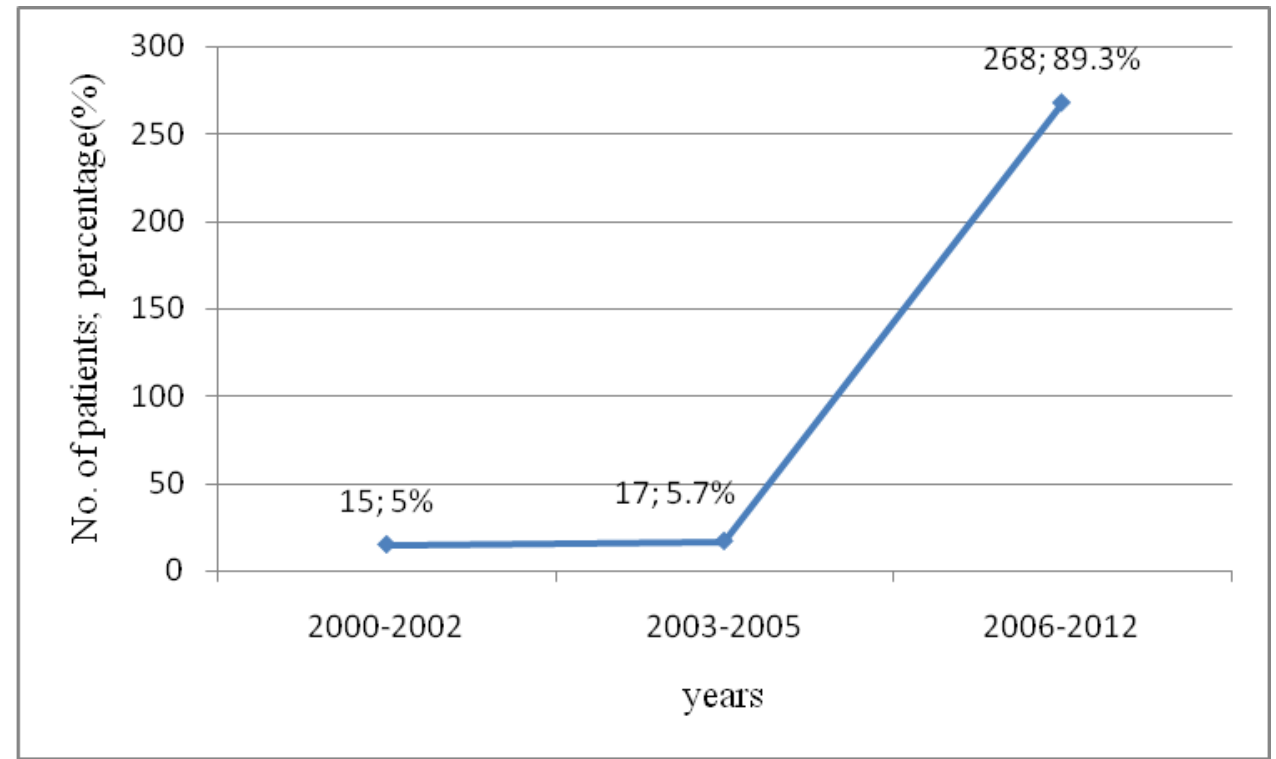

Figure 2: AIDS incidence per year in the sample

Ultrasound findings in liver and biliary tract of AIDS patient are shown in (Table 3 and Figure 3). Out of the study population (88; 29.3\%) presents hepatomegally, $(49 ; 16.3 \%)$ presents portal hypertension, $(20 ; 6.7 \%)$ presents periportal fibrosis, $(42 ; 14 \%)$ increased liver echogenicity; fatty fibrotic changes, $(18 ; 6 \%)$ presents jaundice, $(24 ; 8 \%)$ extra hepatic ducts dilatation, $(33 ; 11 \%)$ thick wall gallbladder; cholecystitis, $(24 ; 8 \%)$ cholelithiasis and (18; $6 \%$ periportal dense bands and small hyperechoic nodules; Kaposi sarcoma.

Table 3: Ultrasound findings in liver and biliary tract of AIDS patients

\begin{tabular}{|l|c|c|}
\hline \multicolumn{1}{|c|}{ Ultrasound findings } & Frequency & Percentage (\%) \\
\hline Hepatomegally & 88 & $(29.3 \%)$ \\
\hline Portal hypertension & 49 & $(16.3 \%)$ \\
\hline Fatty fibrotic changes & 42 & $(14 \%)$ \\
\hline Cholecystitis & 33 & $(11 \%)$ \\
\hline Cholelithiasis & 24 & $(8 \%)$ \\
\hline Extra hepatic ducts dilatation & 24 & $(8 \%)$ \\
\hline Periportal fibrosis & 20 & $(6.7 \%)$ \\
\hline Jaundice & 18 & $(6 \%)$ \\
\hline Kaposi sarcoma & 2 & $(0.7 \%)$ \\
\hline Total & 300 & $100 \%$ \\
\hline
\end{tabular}

Confirmed by liver biopsy; verification by histological laboratory exam. 




Figure 3: Ultrasound findings in the liver and biliary tract of AIDS patients

\section{DISCUSSION}

The prevalence of AIDS out of 300 samples was 198; 66\% males and 102; $34 \%$ females. The scarce data useful for documenting gender differences in HIV prevalence or incidence come from the very few serologic surveys in national populations, and from local, population based studies, most of them longitudinal. It is worth noting that this age pattern of prevalence is similar to that of tuberculosis (more young women, more older men), another lethal persistent infection, which is also a common opportunistic infection for HIV [11-13].

The study reported an increase in the prevalence of AIDS per year in Khartoum state population (Table 2). Understanding the increase prevalence of cases per year was described in base case scenarios, predicted HIV prevalence increases of $24 \%-38 \%$ in 10 years. Reducing the transmission rate by $50 \%$ within 10 years reduces incidence by $40 \%$; prevalence increases $20 \%$ to an estimated 1329000 persons living with HIV. Halving the transmission rate within 5 years reduces incidence by $46 \%$; prevalence increases $13 \%$, to 1247000 . Although in 10 years incidences is similar regardless of the intervention time frame, more infections are averted when halving the transmission rate within 5 years [14]. The back-calculation $(\mathrm{n}=1.230$ million HIV/AIDS cases reported by the end of 2006) yielded an estimate of 55400 (95\% CI, 50 000-60 800) new infections per year for 2003-2006 and indicated that HIV incidence increased in the mid-1990s, then slightly declined after 1999 and has been stable thereafter [15]. In addition, analysis indicates that 571000 new HIV infections occurred in the population 2 years and older during the year 2005 in South Africa [16].

Various diseases associated with human immunodeficiency virus (HIV) infection are often difficult to diagnose. A poor immune response, atypical presentations and opportunistic pathologies all contribute to this difficulty [17]. Imaging plays an important role in the detection of various pathologies associated with AIDS. In many cases, the radiologist is often the first clinician to suspect the possibility of human immunodeficiency virus (HIV) or AIDS in a patient's diagnostic work-up and it is, therefore, important that radiologists are familiar with the imaging features of this disease and its complications [18].

The obtained results reveal wide spectrum of complications in liver and biliary tract related to positive AIDS infection where hepatomegally and cholecystitis are the commonest hepatic and biliary tract complication, such findings was supported by Langer et al. [4] and Uygur et al. [19] where the most common abdominal ultrasound findings in HIV patients were hepatomegally. Dominique et al. [20] reported that cholecystitis is the commonest pathology detected in the biliary tract of positive AIDS patients, which in turn support our findings. 
Ultrasound findings such as cholecystitis and hepatomegally, can be explained in the basis that the most common cause of acute cholecystitis is gallstones, which block the cystic duct. This leads to gallbladder irritation and inflammation. Acalculous cholecystitis, though rare, is most often seen in patients hospitalized in intensive care wards of hospitals. In these cases there are no gallstones. Complications of other diseases, like AIDS or diabetes, cause inflammation. While in hepatomegally, certain viral infections can also result in the liver becoming enlarged, as infections with hepatitis $\mathrm{A}$ or $\mathrm{B}$, infectious mononucelosis and HIV.

\section{CONCLUSION}

In conclusion, abdominal ultrasound is a simple and cost effective tool and can be used as a baseline imaging modality in AIDS infected patients. Its accuracy and sensitivity is invaluable in the assessment of the disease sate and in monitoring of therapy. Liver enlargement and gall bladder wall thickening are common effects develop due to AIDS infection in population at Khartoum state, Sudan. Further studies are required to define patterns of clinical findings, pathologic and laboratory correlates with ultrasound to develop and refine diagnostic algorithms for clinical use.

\section{Funding: Non .}

Conflicts of interest: The authors declare that there is no conflict of interest.

Ethical approval: The protocol of the study was approved by the Ethical Committees of the Radiology and Medical Imaging Departments of Omdurman, Elshaab and Bashayer Teaching Hospitals. Informed consents were obtained from all patients. The procedures followed were in accordance with the ethical standards of the responsible committee on human experimentation (institutional or regional) and with the Helsinki Declaration of 1964.

\section{REFERENCES}

1. Sepkowitz KA. AIDS- The First 20 Years. $N$. Engl. J. Med 2001; 344(23): 1764-1772.

2. Rom WN, Markowitz SB. Environmental and occupational medicine, $4^{\text {th }}$ ed. Lippincott Williams \& Wilkins 2007; 745.

3. Brunetti E, Brigada R, Poletti F, Maiocchi L, Garlaschelli AL, Gulizia R, et al. The Current Role of Abdominal Ultrasound in the Clinical
Management of Patients with AIDS. Ultraschall in Med Journal 2006; 27(1): 20-33.

4. Langer R, Langer M, Schütze B, Wakat JP, Zwicker C, Felix R. Ultrasound findings in patients with AIDS. Digitale Bilddiagn Journal 1988; 8(2): 93-96.

5. Villafae MF, Trione N, Corti M, Mendez N, Gancedo E, Zamora N, et al. Case Report: Primary Liver AIDS Related Lymphoma. Inst. Med. trop. S. Paulo Journal 2006; 48(4): 229231.

6. Millicent $\mathrm{O}$, Mojisola O, Godwin I, Adenike T, Atinuke M, Ademola $\mathrm{J}$, et al. Abdominal Ultrasonography in HIV/AIDS Patients in Southwestern Nigeria. BMC Medical Imaging Journal 2008, 8: 5.

7. Dragica T, Branko B, Djordje J, Brankica D, Milos K, Dubravka S, et al. Liver Enlargement Associated with Opportunistic Infections in Patients with Human Immunodeficiency Virus Infection. Gastrointestin Liver Dis 2008; 17(4): 401-404.

8. Luburich P, Bru C, Ayuso MC, Azón A, Condom E. Hepatic Kaposi sarcoma in AIDS: US and CT Findings. Radiology 1990; 175: 172-174.

9. Palmer PES. Manual of Diagnostic Ultrasound. World Health Organization 1995; 66-69.

10. Gilan SA. Guidelines and Protocols for Medical Diagnostic Ultrasound, $1^{\text {st }}$ ed. Maha Publishing Company 2002; 20-23.

11. Mulder D, Nunn A, Kamali A, Nakiyingi J, Wagner HU, Kengeya Kayondo J. Two Year HIV-1 Associated Mortality in a Ugandan Rural Population. Lancet 1994; 343(8904): 10211023.

12. Kilian AH, Gregson S, Ndyanabangi B, Walusaga K, Kipp W, Sahlmuller G, et al. Reductions in Risk Behavior Provide the Most Consistent Explanation for Declining HIV-1 Prevalence in Uganda. AIDS 1999; 13(3): 391398.

13. Fylkesnes K, Musonda RM, Kasumba K, Ndhlovu Z, Mluanda F, Kaetano L, et al. The HIV Epidemic in Zambia: Socio Demographic Prevalence Patterns and Indications of Trends among Childbearing Women. AIDS 1997; 11(3): $339-45$.

14. Hall HI, Green TA, Wolitski RJ, Holtgrave DR, Rhodes P, Lehman JS, et al. Estimated Future HIV Prevalence, Incidence, and Potential Infections Averted in the United States: A Multiple Scenario Analysis. Acquir Immune Defic Syndr Journal 2010; 55(2): 271-276. 
15. Hall HI, Song R, Rhodes P, Prejean J, An Q, Lee LM, et al. Estimation of HIV Incidence in the United States. Journal AMA 2008;300(5): P: 520529.

16. Rehle T, Shisana O, Pillay V, Zuma K, Puren A, Parker W. National HIV incidence measures new insights into the South African epidemic. $S$ Afr Med Journal 2007; 97: 194-199.

17. Chakraborty P, Bandyopadhyay D. Utility of abdominal ultrasonography in HIV patients. Singapore Med Journal 2009; 50(7): 710
18. Corr P. Imaging of acquired immunodeficiency syndrome (AIDS). Ann Acad Med Singapore Journal 2003; 32: 477-82.

19. Uygur-Bayramicli O, Dabak G, Dabak R. A Clinical Dilemma: Abdominal Tuberculosis. World J Gastroenterol 2003; 9: 1098-1101.

20. Defalque D, Menu Y, Girard PM, Coulaud JP. Sonographic Diagnosis of Cholangitis in AIDS Patients. Abdominal Imaging Journal 1989; 14(1): 143-147. 